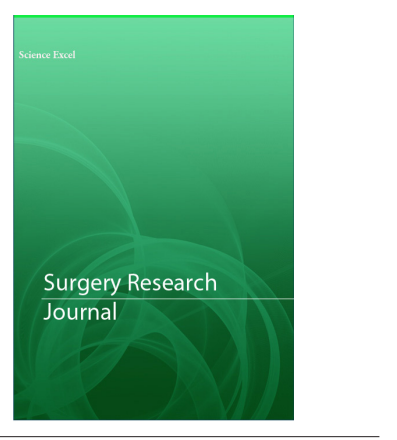

Correspondence

Hani Albadawe

Ministry of the National Guard- Health Affairs, Riyadh, Saudi Arabia

- Received Date: 17 Aug 2021
- Accepted Date: 23 Aug 2021
- Publication Date: 30 Aug 2021

Keywords

sperm retrieval, azoospermia, sperm injections, intracytoplasmic, semen analysis

Copyright

(C) 2021 Science Excel. This is an openaccess article distributed under the terms of the Creative Commons Attribution 4.0 International license.

\title{
Longitudinal vs horizontal capsular incision! Does it alter micro-TESE outcome?
}

\author{
Hani Albadawe', Rizwanul Haque ${ }^{2}$, Naif Alhifthi ${ }^{3}$, Abdullah Almousa ${ }^{4}$, Turki A \\ Alferayan $^{2}$, Khalid A Alrabeeah ${ }^{2,5,6}$, Saad M Abumelha ${ }^{2,5,6}$ \\ 'Johns Hopkins Aramco Healthcare, Dhahran, Kingdom of Saudi Arabia \\ ${ }^{2}$ Ministry of the National Guard- Health Affairs, Riyadh, Saudi Arabia \\ ${ }^{3}$ Security Forces Hospital, Riyadh, Saudi Arabia \\ ${ }^{4}$ Prince Sultan Military Medical City, Riyadh, Saudi Arabia \\ ${ }^{5}$ King Abdullah International Medical Research Center, Riyadh, Saudi Arabia \\ ${ }^{6}$ King Saud bin Abdulaziz University for Health Sciences, Riyadh, Saudi Arabia
}

\begin{abstract}
Micro-TESE (testicular sperm extraction), a procedure performed for treatment of non-obstructive azoospermia (NOA), a leading infertility issue among males globally accounting for about $7 \%$ of male population. Azoospermia is absence of sperms in ejaculate upon semen analysis, $2 \%$ of global population has encountered. A lot is discussed upon sperm retrieval success in both variants of MicroTESE, which are transverse or horizontal and longitudinal or vertical approach, established studies have identified about $45 \%$ to $65 \%$ of success retrieving spermatozoa. The objective being identification of success of both the variants of procedure separately and also of post-operative complications to both approaches for micro-TESE. A cohort study, for which data, secondary (retrospective), was obtained from King Abdulaziz Medical City (KAMC), Riyadh. The time frame for data covered January 2016 to November 2018; 87 patients underwent micro-TESE, as in logbook available in "Best Care System at KAMC. Data obtained was analyzed using SPSS Software, 87 of these patients who underwent micro-TESE procedure, 45 were done with the transverse approach and 42 with longitudinal approach, accounting for $51.7 \%$ and $48.3 \%$, respectively. Upon post-operative evaluation, in transverse approach, sperm were retrieved in $25.29 \%$ and for longitudinal approach retrieval was about $19.54 \%$. The success rate was $48.9 \%$ out of 45 procedures in transverse approach and $40.5 \%$ out of 42 procedures in vertical/ longitudinal approach. The most common reported post-operative complication in transverse type was atrophy $20 \%$ and pain $13.3 \%$ on other hand common complications recorded for vertical type, atrophy was about $17.2 \%$ and pain in $12.6 \%$ of patients. Not much of statistical significance was observed between the transverse and longitudinal approach in either of the outcomes whether it is success rate or post-operative complications. Both approaches are influenced by the factors of surgeon expertise and certain other factors that include pre- and post-operative hormonal therapy, baseline hormonal status and intracytoplasmic sperm injection (ICSI)..
\end{abstract}

\section{Introduction}

Infertility is referred to as couple does not conceive for more than a year of unprotected intercourse. Now it can be both male and female factors, among male factor infertility accounts for about $40-50 \%$ of cases [1], affecting $7 \%$ of all men [2]. It was recorded in previous study conducted in France that there was about $14.1 \%$ of infertility for which about $20 \%$ were male factors [1].

One of those male factors were, Azoospermia, being elaborated as absence of sperm in the ejaculate, upon semen analysis [2]. Approximately 2\% of general population around the globe have azoospermia [3]. Azoospermia is classified into two, one being obstructive azoospermia (OA) and the other non- obstructive azoospermia (NOA), between both, $80 \%$ of azoospermia patients were found to be NOA type. The clinical diagnosis is based on examining the volume and level of follicular stimulating hormone (FSH), prior being small in volume and later with elevated levels are laboratory indicative of azoospermia $[3,4]$. It was a breakthrough on introducing the technique of intracytoplasmic sperm injection (ICSI), in 1992, which has helped couples conceive with male counterparts having NOA [5-8].

Apart from that, a new modality, where it is focused for patient's retrieval of spermatozoa after the procedure, that is testicular sperm extraction (TESE) in other words Microdissection Testicular Sperm Extraction or Micro-TESE. This modality has increased much of success in sperm retrieval in patients with NOA. It was found 
to have retrieved sperms because of spermatozoa presences, by 45 to $63 \%$ of the patients underwent micro-TESE $[8,9]$. This could be well understood by results of a study conducted in 2007 , which recorded about $48 \%$ of success rate, out of 150 patients, for micro-TESE procedure [10].

As in conventional TESE procedure more of tissue damage can occur and tissue loss with possible hematomas and larger scars, but intervention and assisted reproductivity via micro-TESE has drastically decreased that incidences, only 2-10mg samples are obtained which would otherwise had been $50-75 \mathrm{mg}$ via conventional method [11].

As there are two approaches to these procedures, one is transverse approach, in lay terms also called horizontal approach for incision and the other one referred to as longitudinal approach or also sometime called a vertical approach. Most studies have generalized discussion upon the procedure success in countering the male azoospermia type infertility not focusing on individual variants of microTESE, here this study will focus on each of the variants success rate in retrieval of spermatozoa or sperms in the semen, and also will distinguishingly describe postoperative complication following individual procedural approaches.

\section{Methodology}

A retrospective cohort study was done at King Abdulaziz Medical City (KAMC) in Riyadh. Data was collected from January 2016 till November 2018. Patients who underwent micro-TESE procedure for sperm retrieval, in the time frame were included. The data was retrieved from logbook available in "Best care system" at KAMC, of which a sample of 87 was collected.

Collection of the data was in accordance with established questionnaire, which included demographics of patients, procedure related question focusing on pre, intra and postoperative, hematological factors, hormonal status, infections, and testacies volume and other medical records.

In terms of ethical considerations, the respondents were informed and were asked for postop complications and conception, upon their consent and comfort, phone call interviews were held. Their personal information is kept confidential. Records were obtained as per King Abdulaziz Medical City ethical committee approval.

Patients were called and were asked of postoperative complications and conception after they had gone for sperm retrieval via micro-TESE. All the data was analyzed via SPSS

Table 1. Age, BMI, Preoperative Hormonal Assay and Testacies volume.

\begin{tabular}{|c|c|c|c|c|c|c|c|}
\hline & \multirow[t]{2}{*}{ Age } & \multirow[t]{2}{*}{ BMI } & \multicolumn{3}{|c|}{ Preoperative } & \multicolumn{2}{|c|}{ Preop Testacies Volume } \\
\hline & & & FSH & LH & Testosterone & Right & Left \\
\hline Number & \multicolumn{7}{|c|}{87} \\
\hline Mean & 37.78 & 29.22 & 14.96 & 7.81 & 14.01 & 7.42 & 7.54 \\
\hline Median & 36.00 & 29.30 & 13.65 & 6.52 & 12.49 & 7.22 & 7.80 \\
\hline S.D & 8.139 & 5.78 & 10.49 & 6.26 & 9.73 & 5.57 & 5.466 \\
\hline Min. & 25 & 16.00 & .68 & 1.31 & 0.89 & 0.44 & 1.30 \\
\hline Max. & 66 & 41.80 & 40.11 & 33.33 & 48.78 & 28 & 25 \\
\hline
\end{tabular}

BMI: body mass index; FSH: follicle stimuating hormone; LH: luteinizing hormone; SD: standard deviation

Table 2. Frequencies and percentages of Azoospermia types, infertility, Sperm retrieval and surgery type.

\begin{tabular}{|c|c|c|c|}
\hline \multicolumn{4}{|c|}{ Type of Azoospermia } \\
\hline & Frequency & \%Age & Cumulative Percent \\
\hline Non-Obstructive Azoospermia & 64 & $73.6 \%$ & $73.6 \%$ \\
\hline Obstructive Azoospermia & 20 & $23.0 \%$ & $96.6 \%$ \\
\hline Un-Known & 3 & $3.4 \%$ & $100.0 \%$ \\
\hline \multicolumn{4}{|c|}{ Infertility Type } \\
\hline Primary Infertility & 76 & $87.4 \%$ & $87.4 \%$ \\
\hline Secondary Infertility & 11 & $12.6 \%$ & $100.0 \%$ \\
\hline \multicolumn{4}{|l|}{ Sperm Retrieval } \\
\hline Negative & 48 & $55.2 \%$ & $55.2 \%$ \\
\hline Positive & 39 & $44.8 \%$ & $100.0 \%$ \\
\hline \multicolumn{4}{|c|}{ Type of Surgery } \\
\hline Transverse Micro-TESE & 45 & $51.7 \%$ & $51.7 \%$ \\
\hline Longitudinal Micro-TESE & 42 & $48.3 \%$ & $100.0 \%$ \\
\hline Total & 87 & $100.0 \%$ & \\
\hline
\end{tabular}


Table 3. Success Rates of Transverse and Longitudinal Approaches for micro-TESE procedure.

\begin{tabular}{|c|c|c|c|c|c|c|c|c|}
\hline \multirow{3}{*}{$\begin{array}{l}\text { Type of Surgery } \\
\text { \%Ages }\end{array}$} & \multicolumn{4}{|c|}{ Transverse Micro-TESE } & \multicolumn{4}{|c|}{ Longitudinal Micro-TESE } \\
\hline & \multicolumn{3}{|c|}{ Sperm Retrieval } & \multirow{2}{*}{$\begin{array}{c}\text { Total } \\
45 \\
\end{array}$} & \multicolumn{3}{|c|}{ Sperm Retrieval } & \multirow{2}{*}{$\frac{\text { Total }}{42}$} \\
\hline & Positive (success) & \multicolumn{2}{|c|}{ Negative (Failure) } & & Positive (success) & \multicolumn{2}{|c|}{ Negative (Failure) } & \\
\hline & 22 & \multicolumn{2}{|r|}{23} & & 17 & \multicolumn{2}{|r|}{25} & \\
\hline $\begin{array}{l}\text { Success \& Failure Rates } \\
(\% \text { with in procedure })\end{array}$ & $48.9 \%$ (out of 45$)$ & \multicolumn{2}{|c|}{$51.1 \%($ out of 45$)$} & & $40.5 \%$ (out of 42 ) & \multicolumn{2}{|c|}{$59.5 \%$ (out of 42 ) } & \\
\hline Sperm retrieval in total & $25.29 \%$ of 87 cases & \multicolumn{2}{|c|}{$26.4 \%$ of 87 cases } & & $\begin{array}{c}19.54 \% \text { of } 87 \\
\text { cases }\end{array}$ & \multicolumn{2}{|c|}{$\begin{array}{l}28.7 \% \text { of } 87 \\
\text { cases }\end{array}$} & \\
\hline \multicolumn{9}{|c|}{ Total } \\
\hline Positive Sperm Retrieval & \multicolumn{2}{|c|}{ Negative Sperm retrieval } & \multicolumn{2}{|c|}{ Transverse micro-TESE } & \multicolumn{2}{|c|}{ Longitudinal Micro-TESE } & \multicolumn{2}{|l|}{ Sample } \\
\hline $39(44.82 \%)$ & \multicolumn{2}{|l|}{$48(55.17 \%)$} & \multicolumn{2}{|l|}{$45(51.7 \%)$} & \multicolumn{2}{|l|}{$42(48.3 \%)$} & \multicolumn{2}{|l|}{87} \\
\hline
\end{tabular}

Table 4. Chi Square Test and significance of the results as per Pearson Chi-Square Asymptotic Significance for Success rates

\begin{tabular}{|l|c|c|c|}
\hline \multicolumn{4}{|c|}{ Chi-Square Tests } \\
\hline & Value & df & $\begin{array}{c}\text { Asymp. Sig. } \\
(2 \text {-sided) }\end{array}$ \\
\hline Pearson Chi-Square & $9.286 \mathrm{a}$ & 9 & .411 \\
\hline Likelihood Ratio & 11.841 & 9 & .222 \\
\hline $\begin{array}{l}\text { Linear-by-Linear As- } \\
\text { sociation }\end{array}$ & .527 & 1 & .468 \\
\hline N of Valid Cases & 87 & & \\
\hline $\begin{array}{l}\text { a. } 14 \text { cells (70.0\%) have expected count less than 5. The minimum } \\
\text { expected count is .48. }\end{array}$ \\
\hline
\end{tabular}

Table 5. Preop hormonal assay and BMI for Cases with Positive sperm retrieval

\begin{tabular}{|l|l|c|c|c|c|}
\hline \multicolumn{7}{|c|}{ For Positive sperm retrieval } \\
\hline & $\begin{array}{c}\text { Preop } \\
\text { FSH }\end{array}$ & $\begin{array}{c}\text { Preop } \\
\text { Testos- } \\
\text { terone }\end{array}$ & $\begin{array}{c}\text { Preop } \\
\text { LH }\end{array}$ & BMI \\
\hline $\mathrm{N}$ & Valid & 39 & 39 & 39 & 39 \\
\hline & Missing & 0 & 0 & 0 & 0 \\
\hline Mean & 13.7162 & 13.6690 & 6.3121 & 29.4308 \\
\hline Median & 12.2000 & 12.4900 & 5.8800 & 29.4000 \\
\hline Std. Deviation & 9.96533 & 9.56213 & 3.98935 & 5.86449 \\
\hline Minimum & .00 & .00 & .00 & 16.70 \\
\hline Maximum & 32.75 & 36.18 & 15.45 & 41.80 \\
\hline
\end{tabular}

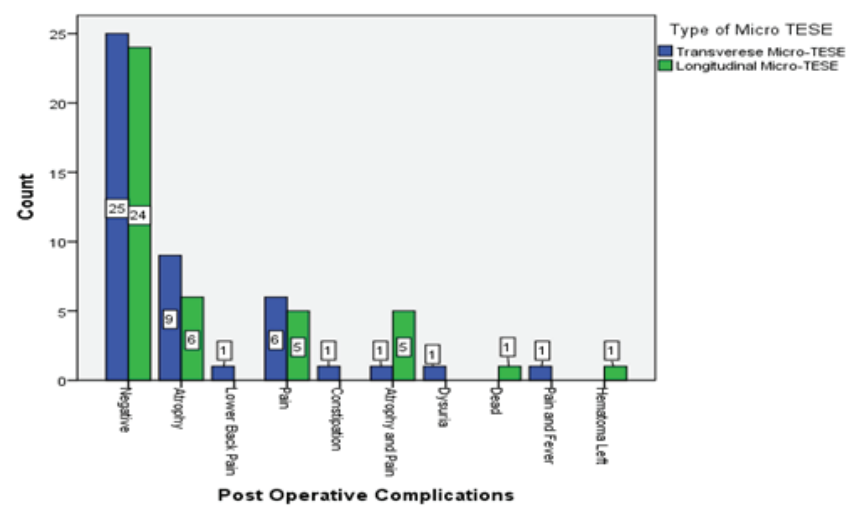

Figure 1. Postoperative complications in both transvers and longitudinal approaches

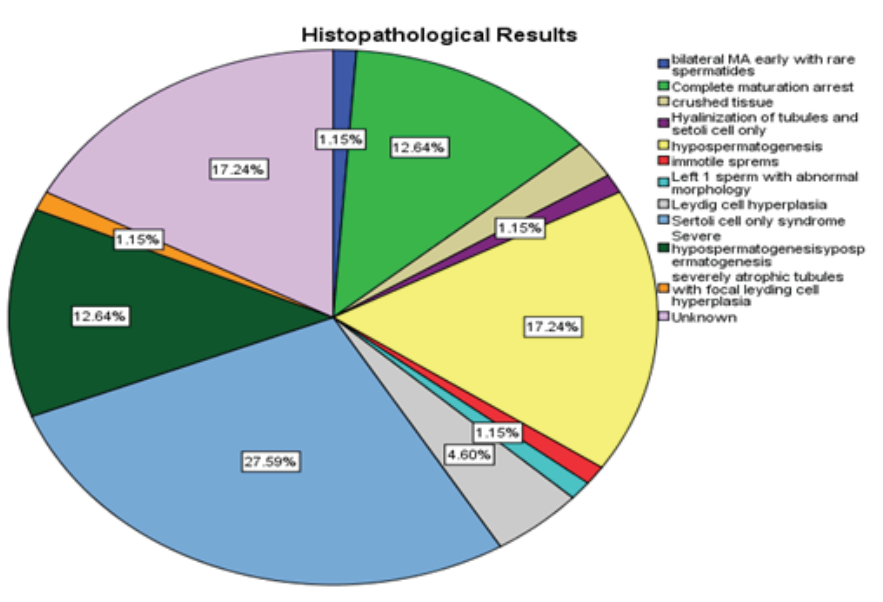

Figure 2. Histopathological Aspects for the patients with Azoospermia 
v 22.0 (IBM Corp.: Armonk, NY, USA) and is presented in tables and graphs of frequencies percentages and as the success rates of separate approaches for sperm retrieval.

\section{Result}

Total of 87 patients were included in the study, each was presented for surgery and underwent micro-TESE for sperm retrieval at National Guard Hospital from January 2016 till November 2018. Mean age observed was 37.78 and body mass index (BMI) in averages of 29.22 with standard deviations of 8.13 and 5.7 respectively. The range for age was $22-66$ and for BMI 16-41.80. (Table 1)

Out of all 87 procedures, 45 were performed using transverse incision technique into the scrotum and 42 counted for longitudinal approach, contributing 51.7\% and 48.3\%, respectively. Total of 64 cases recorded were of NOA type and 20 were having $\mathrm{OA}$, contributing $73.6 \%$ and $23.0 \%$ respectively, this data also includes 3 (about 3.4\%) of the cases which were of unknown type or were not recorded at all. Primary infertility was more prevalent accounting for $87.4 \%$ (76 out of 87 ) in the sample and secondary infertile were about $12.6 \%$ (11 out of 87 ). (Table 2)

The focus being sperm retrieval in this study, results obtained described that overall $44.8 \%$ cases has effectively retrieved, which in numbers are 39 out of 87 cases (Table 2).

For success rate of both variants of micro-TESE in regards with sperm retrieval, it was $48.9 \%$ for those who underwent transverse approach and was $40.5 \%$ for longitudinal approach with significance of 0.411 as per Pearson Chi-square, asymptotic, indicated not much of an effect of procedure on the differential success of sperm retrieval (Tables 3 and 4). In addition, in (Tables 5 and 6) patients with positive sperm retrieval were sharing a mean level and standard deviation of FSH 13.71 \pm 9.9 , luteinizing hormone (LH) 6.31 \pm 3.9 , testosterone $13.66 \pm 9.5$ and BMI 29.4 \pm 5.8 . . In contrast, patients who had negative sperm retrieval were sharing mean level and standard deviation of FSH about $15.97 \pm 10.9$, LH 9.03 \pm 7.4 , testosterone (14.28 \pm 9.9$)$ and BMI $(29.06 \pm 5.7)$.

Lastly, regarding the complication after Micro-TESE (Figure 1 ), showing that the most common reported post-operative complication in transverse type was atrophy $10.34 \%$ and pain $6.90 \%$. In vertical type the most common reported postoperative complication was atrophy about $6.90 \%$ and pain $5.75 \%$ (Figure 1). However, the average pain score in transverse type and in vertical type were $(3.93,4.82)$, respectively. Postoperative complications regarding type of incision results show no statistical significance with a p-value of 0.41 .

The histopathology results with Sertoli cell only syndrome $27.6 \%$, hypospermatogenesis $17.2 \%$ and severe hypospermatogenesis $12.6 \%$ and complete maturation arrest was found in $12.6 \%$. Other results combined made approximately $10.59 \%$ (crushed tissue, immotile sperms, atrophic tubules, hyalinization of tubules and sertoli cell only and leyding cell hyperplasia). 17.2\% were unknown (Figure 2).

\section{Conclusion}

Testicular sperm extraction and intracytoplasmic injection were first introduced in 1993 for the treatment of OA [12], which was widely used for men diagnosed to have NOA [13]. Micro-TESE is considered the gold standard method for surgical sperm retrieval among patients with NOA [14].
Although the technique of micro-TESE is mostly standardized for scrotal and tunica vaginalis incision, but the tunica albuginea incision is still somewhat controversial, as the pioneers of micro-TESE, Dr. Peter Schlegel used to open tunica albuginea by a transverse incision in order to avoid equatorial testicular vessels [14]. The rationale behind it is that sub tunical vessels run horizontally and clearly visible under the microscope, so the risk of bleeding and later on hematoma formation is significantly reduced to near zero.

While a significant number of surgeons still use vertical incision to open the tunica albuginea, as this allows wider exposure to testicular mass and making better access to polar regions of testis.

Both groups have their pros and cons. Originally, Peter Schlegel reported the opening of the tunica albuginea in a transverse plane as a vertical incision will be harmful to the testicular artery [15]. However, Silber noted a wide vertical incision allows extensive visualization of the seminiferous tubule and subtunical vessels [16].

Although the ultimate outcome of micro-TESE is measured in terms of sperm retrieval initially and later on pregnancy rate. Most of the meta-analysis studies for micro-TESE done in the past have results comparing those two goals as the end outcome. Post-procedure complications like hematoma collection and hydrocele are mostly mentioned. The question being success rate of both variants of micro-TESE with respect to sperm retrieval and also post-operative complications, this study is a landmark, as in literature and meta-analysis made no such comparison which is comparing the transverse versus vertical incision of the tunica albuginea for sperm retrieval and post-operative complications.

Although till now no study has compared the sperm retrieval rate with reference to incision of tunica albuginea, this study contradicts the hypothesis that vertical incision has a better sperm retrieval rate versus transverse incision. Other factors that were included were post procedure hematoma formation in both groups, with reference to type of tunical incision. In the vertical incision group only one patient had a hematoma collection, while the transverse group had none. This again support to the rationale that there is less bleeding or vascular injury to sub tunical vessels in transverse incision [15].

\section{Conclusion}

It was obvious that there was not much of significance on accepting which approach is better than other in term of sperm retrieval and postoperative complication specially hematoma or hydrocele formation. This study was done to shed a light on the different techniques and approaches to micro-TESE and it's outcomes. As the sample size is small, further research should be done on a larger scale. As the results have indicated that both have almost close ranged success rate in sperm retrieval and both approaches have much similarities and proportions are in close approximation for postop complications.

\section{Acknowledgments}

We are much grateful to the King Abdulaziz Medical City (KAMC), Riyadh for providing us patient records for the procedure of micro-TESE, and we are much thankful to the participants who willingly consented for providing information when they were called and reached out for collection of data. 


\section{Funding}

Not applicable

\section{Conflict of interest}

No conflict of interests was declared by the authors.

\section{Author's contribution}

Hani Albadawe is the core contributor to the research, the idea and problem identification was presented and discussed among authors. Hani Albadawe worked on data retrieval, development of questionnaire and writing main research report. Rizwanul Haque contributed to Writing the thesis and manuscript for the research, tabulation graphs were also organized by him. Naif Alhfthi, Abdulllah Almousa, Turki A Alferayan, Khalid A Alrabeeah and Saad M Abumelha also contributed to research work and supporting material were provided as in helping with data collection and analysis.

\section{References}

1. Palermo GD, Neri QV, Takeuchi T, Rosenwaks Z. ICSI: where we have been and where we are going. Semin Reprod Med. 2009;27(2):191-201.

2. Jarow JP, Espeland MA, Lipshultz LI. Evaluation of the azoospermic patient. J Urol. 1989;142(1):62-65.

3. Dabaja AA, Schlegel PN. Microdissection testicular sperm extraction: an update. Asian J Androl. 2013;15(1):35-39.

4. Rajfer J. TESA or TESE: Which Is Better for Sperm Extraction?. Rev Urol. 2006;8(3):171.

5. Palermo GD, O'Neill CL, Chow S, et al. Intracytoplasmic sperm injection: state of the art in humans. Reproduction. 2017;154(6):F93-F110.

6. Sullivan EA, Zegers-Hochschild F, Mansour R, et al.
International Committee for Monitoring Assisted Reproductive Technologies (ICMART) world report: assisted reproductive technology 2004. Hum Reprod. 2013;28(5):1375-1390.

7. Amer M, Ateyah A, Hany R, Zohdy W. Prospective comparative study between microsurgical and conventional testicular sperm extraction in non-obstructive azoospermia: follow-up by serial ultrasound examinations. Hum Reprod. 2000;15(3):653-656.

8. Schlegel PN. Testicular sperm extraction: microdissection improves sperm yield with minimal tissue excision. Hum Reprod. 1999;14(1):131-5.

9. Shah R. Surgical sperm retrieval: Techniques and their indications. Indian J Urol. 2011;27(1):102-109.

10. Ramasamy R, Schlegel PN. Microdissection testicular sperm extraction: effect of prior biopsy on success of sperm retrieval. J Urol. 2007;177(4):1447-1449.

11. Janosek-Albright KJC, Schlegel PN, Dabaja AA. Testis sperm extraction. Asian J Urol. 2015;2(2):79-84.

12. Schoysman R, Vanderzwalmen P, Nijs M, et al. Pregnancy after fertilisation with human testicular spermatozoa. Lancet. 1993;342(8881):1237.

13. Silber SJ, Nagy Z, Liu J, et al. The use of epididymal and testicular spermatozoa for intracytoplasmic sperm injection: the genetic implications for male infertility. Hum Reprod. 1995;10(8):20312043.

14. Flannigan R, Bach PV, Schlegel PN. Microdissection testicular sperm extraction. Transl Androl Urol. 2017;6(4):745-52.

15. Schlegel PN, Li PS. Microdissection TESE: sperm retrieval in non-obstructive azoospermia. Hum Reprod Update. 1998;4(4):439.

16. Silber SJ. Microsurgical TESE and the distribution of spermatogenesis in non-obstructive azoospermia. Hum Reprod. 2000;15(11):2278-84. 